The Chittagong Univ. J. Sci. 43(1): 51-66, 2021

\title{
Effects of Phosphate (Pi) on Seed Germination and Seedling Growth of Cucumis melo L. in Arsenic Supplemented Medium
}

\author{
Afroza Hasan Afrin, Bayezid Mahmud Khan and Mohammad Mosharraf Hossain* \\ Institute of Forestry and Environmental Sciences, University of Chittagong, Chittagong-4331, Bangladesh. \\ *Corresponding author: md.mosharraf@ gmail.com
}

Manuscript Submitted on 09/10/2018, Revised manuscript received on 24/05/2021, and accepted on 27/05/2021.

\begin{abstract}
Arsenic (As) is a toxic metalloid widely distributed in the environment posing a serious threat to human health. Enriching the soil with phosphate $(\mathrm{Pi})$ in As condition is believed to reduce the uptake of As in plants. But, it is not clear whether this uptake changes affect the growth behaviors of plant or not. This study, therefore, aimed to determine the efficacy of Pi in balancing the negative effects of As on Cucumis melo as measured in terms of seed germination, seedling growth and biomass production of seedling. The experiment was carried out in petri dish under laboratory conditions in As solution cultures in the absence or presence of Pi. Seeds of $C$. melo were treated with different concentrations (2, 5 and 10ppm) of As, with or without addition of Pi (10ppm). The results revealed that As exposure hindered germination rate, seedling growth and biomass production of seedling to different magnitudes depending on the concentrations. However, addition of $\mathrm{Pi}$ in As solution culture improved the seed germination and seedling growth significantly. Germination were $87 \%$ in T1 (2ppm As), $76 \%$ in T3 (5ppm As) and $72 \%$ in T5 (10ppm As), compared to $90 \%$ in $\mathrm{T} 2$ (2ppm As+10ppm Pi), 82\% in T4 (5ppm As+10ppm Pi) and 77\% in T6 (10ppm As+10ppm $\mathrm{Pi})$. In treatments T1 (2ppm As), T3 (5ppm As) and T5 (10ppm As), seedling dry biomasses were $19.8 \mathrm{mg}, 10.3 \mathrm{mg}$ and $7.7 \mathrm{mg}$ respectively, which were enhanced to $22.1 \mathrm{mg}, 15.9 \mathrm{mg}$ and $12.1 \mathrm{mg}$ respectively in T2 (2ppm As+10ppm Pi), T4 (5ppm As+10ppm Pi) and T6 (10ppm As+10ppm Pi). Similar trend was also recorded for plumule growth, seedling fresh biomass production, seedling vigor, sturdiness etc. Based on the findings, this study recommended the application of Pi to reduce the negative effects of As on C. melo in As solution culture under laboratory condition, that might also be applicable in the field.
\end{abstract}

Keywords: Cucumis melo L., arsenic, phosphate, germination, vigor index, sturdiness.

DOI: https://doi.org/10.3329/cujs.v43i1.57334 
আর্লেনিক একটি বিষাক্ত উপধাতু যা পরিবেশে ব্যাপকভাবে বিস্তৃত এবং মানুযের জন্য স্বাস্য্যহানীকর। ধারণা করা হয় যে, আর্লেনিকযুক্ত মাটিতে ফসফেট প্রয়োগ করা হনে তা গাছের বৃদ্ধিতে আর্লেনিকের নেতিবাচক প্রভাব কমাতে সহায়তা করে। অতএব, Cucumis melo বীজের অঙ্কুরোদগম, চারার বৃদ্ধি এবং চারার জৈবভর উৎপাদনের ক্ষেত্রে আর্সেনিকের নেতিবাচক প্রভাব কমাতে ফসফেটের ভূমিকা নির্ণয় করাই এ গবেষণার উদ্দেশ্য। গবেষণাটি পরীক্ষাগারে পেট্রিডিসে (কাঁচপাত্রে) আর্সেনিকের পানীয় মিশ্রণে সম্পন্ন করা হয়। C. melo এর বীজকে বিভিন্ন ঘনত্বের আর্সেনিক মিশ্রণের ট্রিটমেন্ট দেয়া হয়, যার মধ্যে ফসফেটের উপস্থিতি এবং অনুপস্থিতি রক্ষা করা হয়। ফলাফলে দেখা যায় যে, আর্সেনিক বীজের অঙ্কুরোদগনের হার, চারার বৃদ্ধি এবং চারার জৈবভর উৎপাদনের ক্ষেত্রে নেতিবাচক প্রভাব ফেলে, যদিও এর মাত্রা আর্লেনিকের ঘনত্বের উপর নির্ভরশীল। তবে আর্সেনিকের পানীয় মিশ্রণে ফসফেটের সংযুক্তি বীজের অঙ্কুরোদগম এবং চারার দক্ষতা বাড়াতে তাৎপর্যপূর্ণ ভূমিকা রাখে। অঙ্কুরোদগম, ট্রিটমেন্ট T১ (২ পিপিএম আর্সেনিক) এ ৮৭\%, T৩ (৫ পিপিএম আর্সেনিক) এ ৭৬\%, এবং T৫ (১০ পিপিএম আর্সেনিক) এ ৭২\% ছিল যা বৃদ্ধি পেয়ে ট্রিটমেন্ট T২ (২ পিপিএম আর্সেনিক + ১০ পিপিএম ফসফেট) এ ৯০\%, T8 (৫ পিপিএম আর্সেনিক + ১০ পিপিএম ফসফেট) এ b-২\% এবং T৬ (১০ পিপিএম আর্সেনিক + ১০ পিপিএম ফসফেট) এ ৭৭\% হয়েছিল। অন্যদিকে, ট্রিটনেন্ট T১ (২ পিপিএম আর্সেনিক), T৩ (৫ পিপিএম আর্সেনিক) এবং T৫ (১০ পিপিএম আর্সেনিক) এ চারার শ্কস্ক-জৈবভর ছিল যথাক্রমে ১৯.৮- মিলিগ্রাম, ১০.৩ মিলিগ্রাম এবং ৭.৭ মিলিগ্রাম, যা বৃদ্ধি পেয়ে ট্রিটন্নেন T2 (২ পিপিএম আর্সেনিক + ১০ পিপিএম ফসফেট), T8 (৫ পিপিএম আর্সেনিক + ১০ পিপিএম ফসফেট) এবং T৬ (১০ পিপিএম আর্সেনিক + ১০ পিপিএম ফসফেট) এ যথাক্রমে ২২.১ মিলিগ্রাম, ১৫.৯ মিলিগ্রাম এবং ১২.১ মিলিগ্রাম হয়েছিল। কান্ডের বৃদ্ধি, চারার আদ্র-জৈবভর উৎপাদন, চারার জীবনিশক্তির ক্ষেত্রেও একই প্রবণতা লক্ষ্য করা গিয়েছিল। প্রাপ্ত ফলাফল বিশ্লেষণপূর্বক গবেষণাকর্মটি থেকে এই সুপারিশ করা যায় যে, পরীক্ষাগারে আর্লেনিকের পানীয় মিশ্রণে C. melo এর বীজের অঙ্কুরোদগম, চারার বৃদ্ধির ক্ষেত্রে আর্সেনিক যে নেতিবাচক প্রভাব ফেলে তা কমিয়ে আনতে ফসফেট প্রয়োগ করা যেতে পারে। একই ধরণের গবেযণা মাঠ-পর্যাঢয় বাস্তবায়ন করা যেতে পারে ।

\section{Introduction}

Native to Central Asia with uncultivated indigenous relatives in tropical and subtropical Africa - Muskmelon (Cucumis melo L.) belongs to the horticulturally diverse gourd family Cucurbitaceae. For their juicy, edible, often musky-scented flesh, melons are popular as a crop in warm countries worldwide including Bangladesh [1]. Accordingly, melons have evolved into several varieties of which $C$. melo is rich in 
Effects of Phosphate (Pi) on Seed Germination and Seedling Growth of Cucumis melo L. in Arsenic 53 Supplemented Medium

bioactive contents including ascorbic acid, carotene, folic acid, and potassium [2]. Well-drained, sandy loam soils having a $\mathrm{pH}$ between 6.0 and 6.5 best suits $C$. melo growth with proper irrigation [3].

Unfortunately, in Bangladesh, elevated concentration of arsenic (As) in surface soil and in plant tissue were reported since As contaminated groundwater has been used for irrigation [4]. Areas under melon cultivation in Bangladesh are prone to the risk. Being a toxic element, As poses serious threats to environment and human health [5-9]. When crops are grown in As-contaminated soil or irrigated with Ascontaminated water, As gets accumulated in the seeds or grain which is becoming an increasingly important problem in many parts of the world [10] as it poses significant risk to animal and human health through soil-crop transfer [11, 12]. At high level of As concentrations, biomass production and yields of a variety of crops shrink significantly [13, 14]. Especially, plants with highly water-retentive fruits, such as melons, accumulates As in elevated concentrations in their fruit [15]. Muskmelon, however, is naturally efficient in preventing As accumulation in the fruit [16].

On the other hand, Phosphate $(\mathrm{Pi})$ - an essential plant nutrient that regulates cellular energy transfer, respiration, and photosynthesis [17] is a chemical analogue of As $[18,19,20]$. Therefore, during uptake of nutrients in plant, Pi contests As due to their chemical similarity [21]. Consequently, the changes in P transport mechanisms regulates As uptake [22] and evidently, the use of $\mathrm{Pi}$ as a fertilizer modifies the sorption/desorption of As in soil environment [23]. Although, it is believed that enriching the soil with $\mathrm{Pi}$ in As condition reduces the uptake of As in plants [5, 24, 25], but it is not clear whether this uptake changes affect the growth behaviors of plant or not. Therefore, this in-vitro study was carried out aiming to determine the efficacy of $\mathrm{Pi}$ in balancing the negative effects of As on $C$. melo with respect to seed germination, seedling growth and biomass production. 
54 Afroza Hasan Afrin, Bayezid Mahmud Khan and Mohammad Mosharraf Hossain

\section{Materials and Methods}

\subsection{Study site and period of study}

The experiment was carried out at the environmental lab of the Institute of Forestry and Environmental sciences $\left(91^{\circ} 50^{\prime} \mathrm{E}\right.$ and $\left.22^{\circ} 30^{\prime} \mathrm{N}\right)$, University of Chittagong (IFESCU), Bangladesh. It was conducted between January and May 2018 as the seeds of C. melo are mostly available in this period in Bangladesh.

\subsection{Collection of seeds}

Healthy and disease-free seeds were extracted from the ripen C. melo fruits. Seeds were dried in the sunlight before storage in airtight polybags which were then kept in refrigerator until use. Seeds of uniform size and color were selected to avoid nontreatment variations [26]. Seeds were tested for viability by using water floating method before use.

\subsection{Preparation of Hoagland's and other solutions}

Hoagland's nutrient solution was prepared as stated in Hoagland and Arnon [27]. The solution comprised of $\mathrm{KNO}_{3}, 0.5 \mathrm{~g} \mathrm{~L}^{-1} ; \mathrm{Ca}\left(\mathrm{NO}_{3}\right)_{2} .4 \mathrm{H}_{2} \mathrm{O}, 1.2 \mathrm{~g} \mathrm{~L}^{-1}$; $\mathrm{MgSO}_{4} .7 \mathrm{H}_{2} \mathrm{O}, 0.5 \mathrm{~g} \mathrm{~L}^{-1} ; \mathrm{H}_{3} \mathrm{BO}_{3}, 2.8 \mathrm{mg} \mathrm{L}^{-1} ; \mathrm{ZnSO}_{4}, 0.2 \mathrm{mg} \mathrm{L}^{-1} ; \mathrm{CuSO}_{4}, 0.05 \mathrm{mg} \mathrm{L}^{-1}$; $\mathrm{NH}_{4} \mathrm{NO}_{3}, 0.08 \mathrm{mg} \mathrm{L}^{-1} ; \mathrm{MnCl}_{2} .4 \mathrm{H}_{2} \mathrm{O}, 1.8 \mathrm{mg} \mathrm{L}^{-1} ; \mathrm{Na}_{2} \mathrm{MoO}_{4} .2 \mathrm{H}_{2} \mathrm{O}, 0.12 \mathrm{mg} \mathrm{L}^{-1}$; FeEDTA, $0.02 \mathrm{~g} \mathrm{~L}^{-1}$; in a volume of $1 \mathrm{~L}$. Pi was added as $0.07 \mathrm{~g} \mathrm{~L}^{-1}$ of $\mathrm{KH}_{2} \mathrm{PO}_{4}$ for Pi treatment and As was added as $0.042 \mathrm{~g} \mathrm{~L}^{-1}$ of $\mathrm{Na}_{2} \mathrm{HAsO}_{4} .7 \mathrm{H}_{2} \mathrm{O}$ for As treatment. The stock solutions were diluted as required for various treatments. The $\mathrm{pH}(6.0)$ of the stock and diluted solutions was adjusted with $1 \mathrm{M} \mathrm{HCl}$ and $1 \mathrm{M} \mathrm{NaOH}$ for all the treatments. Hoagland's solution without $\mathrm{Pi}$ was common for all the treatments including control.

\subsection{Experimental design and treatment combinations}

Petri dishes were sterilized by keeping overnight at $200^{\circ} \mathrm{C}$ in convection oven. In each petri dish, three layers of moist sterilized filter paper were placed. A Randomized Complete Block Design (RCBD) with 7 treatments with 5 replications was adopted for this experiment. A total of 35 petri dishes were needed for this experiment. The treatment combinations used in the experiment were: $\mathrm{T} 0=$ Control (Hoagland's solution without $\mathrm{Pi}$, which was also common for all other treatments); 
$\mathrm{T} 1=2 \mathrm{ppm} \mathrm{As} ; \mathrm{T} 2=2 \mathrm{ppm} \mathrm{As}+10 \mathrm{ppm} \mathrm{Pi} ; \mathrm{T} 3=5 \mathrm{ppm} \mathrm{As} ; \mathrm{T} 4=5 \mathrm{ppm} \mathrm{As}+10 \mathrm{ppm}$ Pi; T5 = 10ppm As; T6 = 10ppm As + 10ppm Pi.

Seeds were soaked in $0.05 \%$ Mercuric chloride solution for 1 minute for sterilization followed by washing with distilled water and drying before sowing them on petri dishes. In each petri dish, 20 seeds of $C$. melo were sown and a total of seven hundred seeds were subjected to 7 different treatments. After sowing the seeds, all the petri dishes were placed in the incubator at $25 \pm 2{ }^{\circ} \mathrm{C}$ temperature. The filter papers of the petri dishes were kept constantly wet at the same level by applying the specific solution of As and $\mathrm{Pi}$.

\subsection{Data recording}

Germination was recorded daily from the date of seed sowing to the last of germination. The seedlings were let to grow for two weeks from the time of seed sowing. After two weeks, five representative seedlings from each treatment were selected to measure growth parameters. The recorded parameters were plumule and radical lengths, collar diameter, fresh weights of plumule and radicle, dry weights of plumule and radicle, number of lateral roots. Plumule and radical were oven dried at $75^{\circ} \mathrm{C}$ for $48 \mathrm{hr}$ to record dry weights. Total height from the collar area to seedling tip of each seedling in each petridish was measured using a ruler. Vigor index was calculated according to Abdul-Baki and Anderson [28] as germination percent $x$ mean total length of seedling i.e. total of shoot and root lengths. Volume index was obtained by multiplying plumule length $(\mathrm{cm})$ with the square of collar diameter $(\mathrm{mm})^{2}$ of the seedlings [29]. Sturdiness was obtained by dividing of plumule length $(\mathrm{cm})$ with collar diameter $(\mathrm{cm})$ of the seedling. Quality index to quantify seedlings morphological quality was calculated following Dickson et al. [30]. The formula for calculating quality index is as follow:

$$
Q I=\frac{T_{d w}}{\left(\frac{H}{D_{c}}+\frac{S_{d w}}{R_{d w}}\right)}
$$


where, QI = Quality index, $\mathrm{T}_{\mathrm{dw}}=$ Total dry weight $(\mathrm{g}), \mathrm{H}=$ Seedling height or plumule length $(\mathrm{cm}), \mathrm{D}_{\mathrm{c}}=$ Collar diameter $(\mathrm{mm}), \mathrm{S}_{\mathrm{dw}}=$ Shoot/plumule dry weight $(\mathrm{g})$, $\mathrm{R}_{\mathrm{dw}}=$ Root/radicle dry weight $(\mathrm{g})$.

\subsection{Statistical analysis}

The SPSS ver.23 was used for statistical analysis of data related to seed germination and seedling growth attributes. The statistical significance $(p<0.05)$ of the differences among the mean values was ascertained by Duncan's multiple range test (DMRT). Different letters in the table indicates significant differences.

\section{Results and Discussion}

\subsection{Germination percentage}

Germination were $87 \%$ in T1 (2ppm As), $76 \%$ in T3 (5ppm As) and $72 \%$ in $\mathrm{T} 5$ (10ppm As), while they were 90\% in T2 (2ppm As+10ppm Pi), 82\% in T4 (5ppm As+10ppm Pi) and 77\% in T6 (10ppm As+10ppm Pi). The results denoted that as the concentration of As increased in solution culture, the germination of seed decreased. However, the addition of $\mathrm{Pi}$ at $10 \mathrm{ppm}$ in As solution culture enhanced the seed germination of $C$. melo and reduced the negative of As (Table 1).

\subsection{Mean daily and cumulative germination percentages}

The highest mean daily germination percent was $45 \%$ in T2 (2ppm As +10 ppm Pi) on the $3^{\text {rd }}$ day followed by $44 \%$ in T4 (5ppm As+10ppm Pi) on the $2^{\text {nd }}$ day (Fig. 1). The highest cumulative germination percent from $3^{\text {rd }}$ day up to the $6^{\text {th }}$ day was in $\mathrm{T} 2$ $\left(2 \mathrm{ppm}\right.$ As $+10 \mathrm{ppm} \mathrm{Pi}$ ) while, from $7^{\text {th }}$ day up to the $8^{\text {th }}$ day, it was the highest in $\mathrm{T} 0$ (Control). The lowest cumulative germination was recorded in T5 (10 ppm As) (Fig. 2). The findings indicated the negative effects of As on the progress of seed germination of $C$. melo with respect to time. The findings also indicated that with the addition of $\mathrm{Pi}$ in As solution culture helped to minimize the negative effects of As on the progress of seed germination. 
Effects of Phosphate (Pi) on Seed Germination and Seedling Growth of Cucumis melo L. in Arsenic 57 Supplemented Medium

Table 1. Phosphate $(\mathrm{Pi})$ on seed germination, plumule and radical growth, collar diameter and number of lateral root production of Cucumis melo in arsenic (As) condition.

\begin{tabular}{|c|c|c|c|c|c|c|c|c|c|c|}
\hline \multirow[t]{2}{*}{ Treatment } & \multirow{2}{*}{$\begin{array}{c}\text { Germination } \\
(\%)\end{array}$} & \multicolumn{2}{|c|}{ Length (cm) } & \multirow{2}{*}{$\begin{array}{l}\text { Plumule- } \\
\text { radicle } \\
\text { ratio }\end{array}$} & \multirow{2}{*}{$\begin{array}{l}\text { Total } \\
\text { length } \\
(\mathrm{cm})\end{array}$} & \multirow{2}{*}{$\begin{array}{c}\text { Collar } \\
\text { diameter } \\
(\mathrm{mm})\end{array}$} & \multirow{2}{*}{$\begin{array}{c}\text { Number } \\
\text { of lateral } \\
\text { root }\end{array}$} & \multicolumn{3}{|c|}{ Increased/decreased (\%) } \\
\hline & & Plumule & Radicle & & & & & Germination & $\begin{array}{l}\text { Total } \\
\text { length }\end{array}$ & $\begin{array}{c}\text { Lateral } \\
\text { root }\end{array}$ \\
\hline T0 & $91_{a}$ & $6.7_{\mathrm{a}}$ & $5.4_{a}$ & $1.24 \mathrm{~d}$ & $12.1_{\mathrm{a}}$ & $1 . \mathrm{a}_{\mathrm{a}}$ & $19 \mathrm{a}$ & 0.0 & 00.0 & 0.0 \\
\hline $\mathrm{T} 1$ & $87 \mathrm{a}$ & $5.0_{\mathrm{bc}}$ & $2.4_{\mathrm{c}}$ & $2.08 \mathrm{~b}$ & $7.4_{b}$ & $1.6_{\mathrm{ab}}$ & $16_{\mathrm{abc}}$ & -4.4 & -38.9 & -15.8 \\
\hline $\mathrm{T} 2$ & $90 \mathrm{a}$ & $6.3_{\mathrm{ab}}$ & $4.5_{b}$ & $1.40 \mathrm{c}$ & $10.8_{\mathrm{a}}$ & $1.7_{\mathrm{ab}}$ & $18_{\mathrm{ab}}$ & -1.1 & -10.7 & -5.3 \\
\hline $\mathrm{T} 3$ & $76_{\mathrm{cd}}$ & $4.0_{\mathrm{c}}$ & $1.2 \mathrm{~d}$ & $3.33 \mathrm{a}$ & $5.2_{\mathrm{c}}$ & $1.5_{\mathrm{ab}}$ & $10_{\mathrm{cd}}$ & -16.5 & -57.0 & -47.4 \\
\hline $\mathrm{T} 4$ & $82_{b}$ & $5.0_{\mathrm{bc}}$ & $2.5_{\mathrm{c}}$ & $2.00 \mathrm{~b}$ & $7.5_{b}$ & $1.4_{\mathrm{ab}}$ & $12_{\text {bed }}$ & -9.9 & -38.0 & -36.8 \\
\hline T5 & $722_{d}$ & $1.9_{\mathrm{d}}$ & $1.0_{\mathrm{d}}$ & $1.90 \mathrm{c}$ & $2.9_{\mathrm{d}}$ & $1.3_{\mathrm{b}}$ & $08_{d}$ & -20.9 & -76.0 & -57.9 \\
\hline T6 & $77_{\mathrm{c}}$ & $4.1_{\mathrm{c}}$ & $1.5_{\mathrm{d}}$ & $2.73 \mathrm{a}$ & $5.6_{\mathrm{c}}$ & $1.4_{\mathrm{ab}}$ & $10_{\mathrm{cd}}$ & -15.4 & -53.7 & -47.4 \\
\hline$P$ value & $<0.001$ & $<0.001$ & $<0.001$ & $<0.001$ & $<0.001$ & 0.115 & 0.012 & -- & -- & -- \\
\hline$F$ value & 23.16 & 14.14 & 71.74 & 35.62 & 27.81 & 2.13 & 4.27 & -- & -- & - \\
\hline
\end{tabular}

$\mathrm{a}-\mathrm{d}=$ Mean values with different lowercase subscripts in a column indicates significant difference at $p<0.05$ according to Duncan's Multiple Range Test (DMRT). T0 $=$ Control, T1=2ppm As, T2 $=2 \mathrm{ppm}$ As $+10 \mathrm{ppm} \mathrm{Pi}, \mathrm{T} 3=5 \mathrm{ppm}$ As, T4= 5ppm As $+10 \mathrm{ppm} \mathrm{Pi}, \mathrm{T} 5=10 \mathrm{ppm}$ As, T6= 10ppm As $+10 \mathrm{ppm}$ Pi.

\subsection{Growth performance}

Plumule lengths were $5.0 \mathrm{~cm}, 4.0 \mathrm{~cm}$ and $1.9 \mathrm{~cm}$, respectively in treatments $\mathrm{T} 1(2 \mathrm{ppm}$ As), T3 (5ppm As) and T5 (10ppm As), while they were $6.3 \mathrm{~cm}, 5.0 \mathrm{~cm}$ and $4.1 \mathrm{~cm}$ in respective treatments T2 (2ppm As+10ppm Pi), T4 (5ppm As+10ppm Pi) and T6 (10ppm As+10ppm Pi). The outcome clearly demonstrated that As at different concentrations in solution culture reduced the plumule growth of $C$. melo while the addition of $\mathrm{Pi}$ at $10 \mathrm{ppm}$ in corresponding As solution cultures minimized the adverse effects of As and enhanced the plumule growth (Table 1). Significantly $(p<0.05)$ high $(5.4 \mathrm{~cm})$ and low $(1.0 \mathrm{~cm})$ radical growths were recorded respectively in T0 (Control) and in T5 (10ppm As). In treatments T1 (2ppm As), T3 (5ppm As) 
and T5 (10ppm As), the number of lateral roots were 16, 10 and 8 respectively, while they were 18, 12 and 10 respectively in T2 (2ppm As+10ppm Pi), T4 (5ppm As+10ppm Pi) and T6 (10ppm As+10ppm Pi). Similar trend was also observed for collar diameter (Table 1).

Table 2. Fresh and dry biomasses, vigor index and volume index of Cucumis melo in arsenic (As) solution cultures under phosphate $(\mathrm{Pi})$ amendment.

\begin{tabular}{cccccccc}
\hline Treatment & \multicolumn{2}{c}{ Seedling } & \multicolumn{2}{c}{ Index } & \multicolumn{2}{c}{ Increased/decreased (\%) } \\
& biomass $(\mathbf{m g})$ & & & & & \\
\cline { 2 - 8 } & Fresh & Dry & Vigor & Volume & Dry & $\begin{array}{c}\text { Vigor } \\
\text { index }\end{array}$ & $\begin{array}{c}\text { Volume } \\
\text { index }\end{array}$ \\
\hline T0 & $178_{\mathrm{a}}$ & $25.4_{\mathrm{a}}$ & $1101_{\mathrm{a}}$ & $21.7_{\mathrm{a}}$ & 00.0 & 0.0 & 0.0 \\
T1 & $129_{\mathrm{c}}$ & $19.8_{\mathrm{bc}}$ & $644_{\mathrm{b}}$ & $12.8_{\mathrm{abc}}$ & -22.1 & -41.5 & -41.0 \\
T2 & $157_{\mathrm{b}}$ & $22.1_{\mathrm{ab}}$ & $972_{\mathrm{a}}$ & $18.2_{\mathrm{ab}}$ & -13.1 & -11.7 & -16.1 \\
T3 & $72_{\mathrm{f}}$ & $10.3_{\mathrm{ef}}$ & $395_{\mathrm{de}}$ & $9.0_{\mathrm{bcd}}$ & -59.5 & -64.1 & -58.5 \\
T4 & $112_{\mathrm{d}}$ & $15.9_{\mathrm{cd}}$ & $615_{\mathrm{bc}}$ & $9.8_{\mathrm{bcd}}$ & -37.3 & -44.1 & -54.8 \\
T5 & $53_{\mathrm{g}}$ & $7.7_{\mathrm{f}}$ & $209_{\mathrm{e}}$ & $3.2_{\mathrm{d}}$ & -69.6 & -81.0 & -85.3 \\
T6 & $85_{\mathrm{e}}$ & $12.1_{\mathrm{de}}$ & $431_{\mathrm{cd}}$ & $8.0_{\mathrm{cd}}$ & -52.3 & -60.9 & -63.1 \\
\hline $\boldsymbol{P}$ value & $<0.001$ & $<0.001$ & $<0.001$ & 0.009 & -- & -- & -- \\
\hline $\boldsymbol{F}$ value & 846.12 & 23.25 & 25.09 & 4.58 & -- & -- & -- \\
\hline
\end{tabular}

a-g = Mean values with different lowercase subscripts in a column indicates significant difference at $p<0.05$, according to Duncan's Multiple Range Test (DMRT). T0 $=$ Control, T1 $=2 \mathrm{ppm}$ As, $\mathrm{T} 2=2 \mathrm{ppm}$ As $+10 \mathrm{ppm} \mathrm{Pi}, \mathrm{T} 3=5 \mathrm{ppm}$ As, $\mathrm{T} 4=$ $5 \mathrm{ppm}$ As $+10 \mathrm{ppm} \mathrm{Pi}, \mathrm{T} 5=10 \mathrm{ppm}$ As, T6 $=10 \mathrm{ppm}$ As $+10 \mathrm{ppm}$ Pi. 
Effects of Phosphate (Pi) on Seed Germination and Seedling Growth of Cucumis melo L. in Arsenic 59 Supplemented Medium

\subsection{Biomass production}

Seedling fresh biomasses were $129 \mathrm{mg}, 72 \mathrm{mg}$ and $53 \mathrm{mg}$ respectively in treatments $2 \mathrm{ppm}$ As, $5 \mathrm{ppm}$ As and $10 \mathrm{ppm}$ As. They were $157 \mathrm{mg}, 112 \mathrm{mg}$ and $85 \mathrm{mg}$ respectively in $2 \mathrm{ppm}$ As $+10 \mathrm{ppm} \mathrm{Pi,} 5 \mathrm{ppm}$ As $+10 \mathrm{ppm} \mathrm{Pi}$ and $10 \mathrm{ppm}$ As +10 ppm Pi. It was $178 \mathrm{mg}$ in control treatment. On the other hand, seedling dry biomasses were $19.8 \mathrm{mg}, 10.3 \mathrm{mg}$ and $7.7 \mathrm{mg}$ respectively, in treatments $\mathrm{T} 1(2 \mathrm{ppm}$ As), T3 (5ppm As) and T5 (10ppm As). They were $22.1 \mathrm{mg}, 15.9 \mathrm{mg}$ and $12.1 \mathrm{mg}$ respectively in T2 (2ppm As+10ppm Pi), T4 (5ppm As+10ppm Pi) and T6 (10ppm As+10ppm Pi) (Table 2).

\subsection{Vigor, volume and quality indices, and sturdiness}

Vigor index were 644 in T1 (2ppm As), 395 in T3 (5ppm As) and 209 in T5 (10ppm As), while they were 972, 615 and 431 respectively in T2 (2ppm As+10ppm Pi), T4 (5ppm As+10ppm Pi) and T6 (10ppm As+10ppm Pi) as shown in Table 2 and the differences were significant at $P<0.05$. In treatments T1 (2ppm As), T3 (5ppm As) and T5 (10ppm As), volume index were 12.8, 9.0 and 3.2 in T1 (2ppm As), T3 (5ppm As) and T5 (10ppm As) respectively compared to 18.2, 9.8 and 8.0 respectively in T2 (2ppm As+10ppm Pi), T4 (5ppm As+10ppm Pi) and T6 (10ppm As+10ppm Pi). Quality index values, as shown in Fig. 3, were 0.0030 in T1 (2ppm As), 0.0010 in $\mathrm{T} 3(5 \mathrm{ppm} \mathrm{As})$ and 0.0008 in T5 (10ppm As) in contrast with significantly $(p<0.05)$ higher $0.0037,0.0021$ and 0.0014 respectively in T2 $(2 \mathrm{ppm}$ As+10ppm Pi), T4 (5ppm As+10ppm Pi) and T6 (10ppm As+10ppm Pi). The highest quality index value was 0.0049 for T0 (control). In treatments T1 (2ppm As), T3 (5ppm As) and T5 (10ppm As), sturdiness were 31.3, 26.7 and 14.6 respectively compared to significantly higher $(p<0.05)$ respective values 37.135 .729 .3 in T2 (2ppm As+10ppm Pi), T4 (5ppm As+10ppm Pi) and T6 (10ppm As+10ppm Pi) (Fig. 4). The observations showed that, along with the seed germination and seedling growth, As also reduced the vigor index, volume index, quality index and sturdiness of $C$. melo and the rate of reduction increased with the increase of the concentration of As in solution culture. However, addition of $\mathrm{P}$ at $10 \mathrm{ppm}$ in As solution culture 
helped to minimize the negative effects of As and enhanced the vigor index, volume index, quality index and sturdiness of this species.

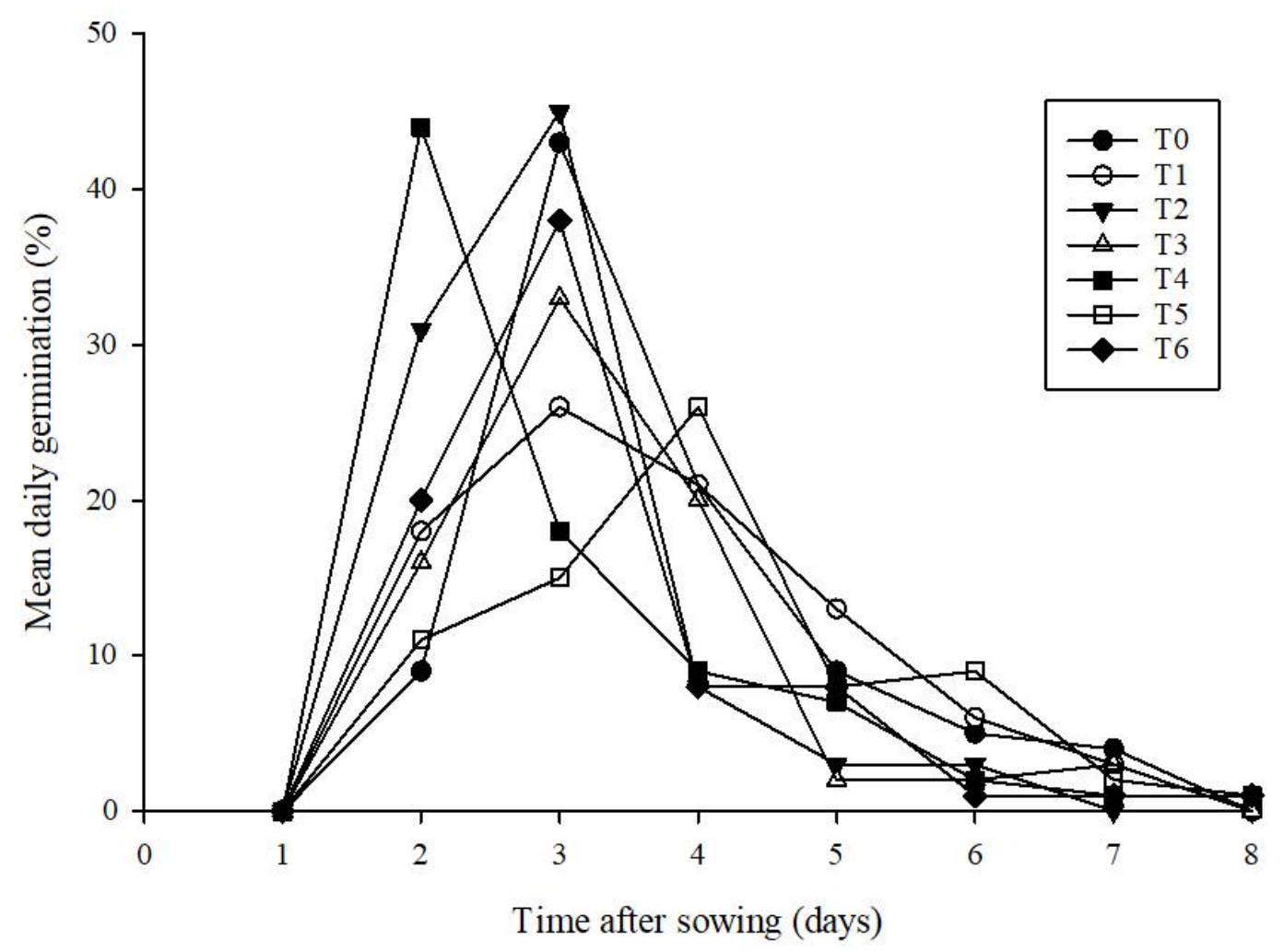

Figure 1: Impacts of phosphate (Pi) on mean daily germination (\%) of Cucumis melo seeds in arsenic (As) condition. $\mathrm{T} 0=$ Control, $\mathrm{T} 1=2 \mathrm{ppm} \mathrm{As}, \mathrm{T} 2=2 \mathrm{ppm} \mathrm{As}+$ $10 \mathrm{ppm} \mathrm{Pi}, \mathrm{T} 3=5 \mathrm{ppm}$ As, T4 $=5 \mathrm{ppm}$ As $+10 \mathrm{ppm} \mathrm{Pi}$, T5 $=10 \mathrm{ppm} \mathrm{As}$, T6 $=10 \mathrm{ppm}$ As $+10 \mathrm{ppm}$ Pi. 
Effects of Phosphate (Pi) on Seed Germination and Seedling Growth of Cucumis melo L. in Arsenic Supplemented Medium

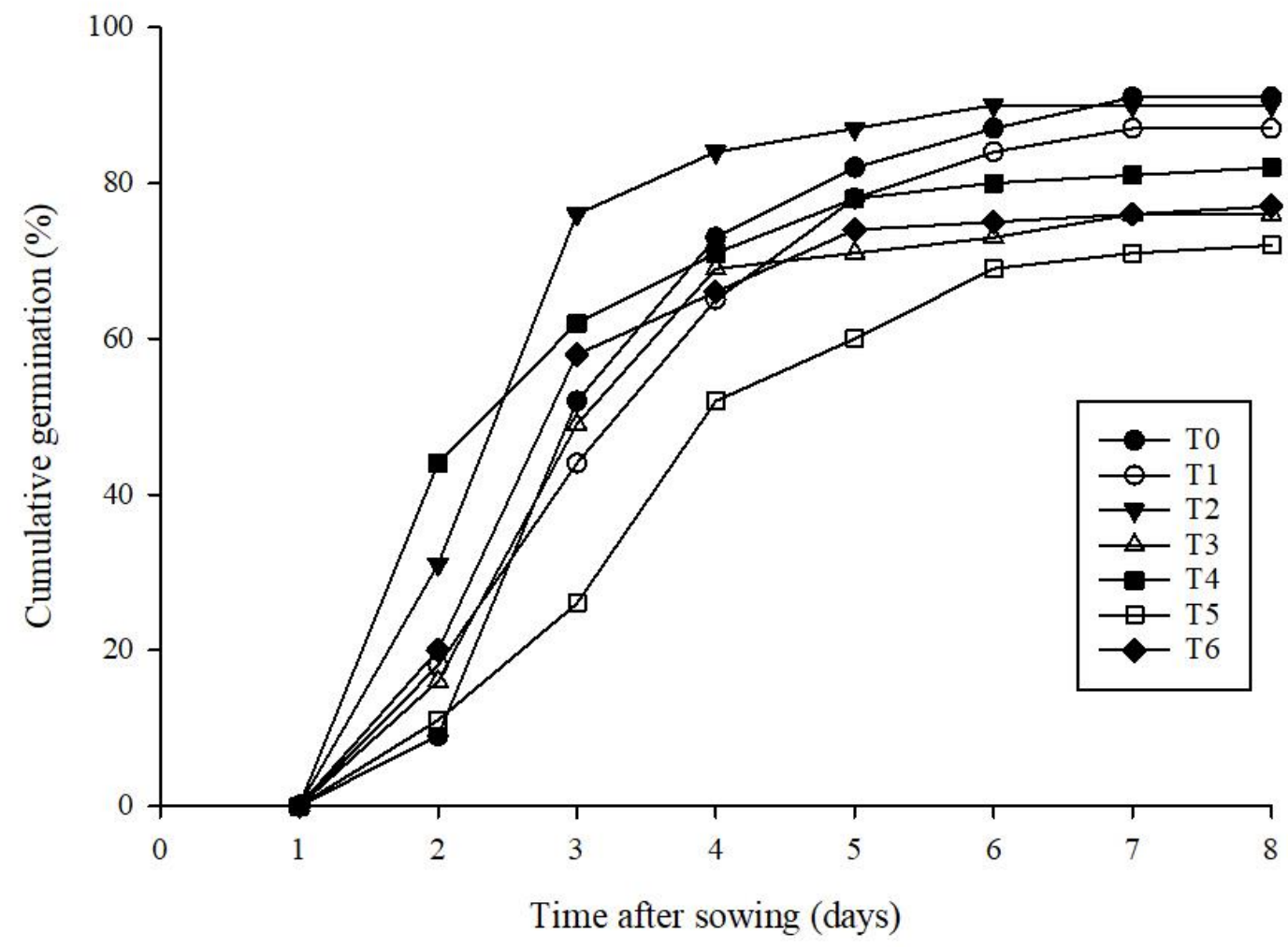

Figure 2: Cumulative germination (\%) of Cucumis melo seeds in arsenic (As) condition under phosphate $(\mathrm{Pi})$ amendment. $\mathrm{T} 0=$ Control, $\mathrm{T} 1=2 \mathrm{ppm}$ As, $\mathrm{T} 2=2 \mathrm{ppm}$ As $+10 \mathrm{ppm} \mathrm{Pi}, \mathrm{T} 3=5 \mathrm{ppm}$ As, T4 $=5 \mathrm{ppm}$ As $+10 \mathrm{ppm}$ Pi, T5= 10ppm As, T6= $10 \mathrm{ppm}$ As + 10ppm Pi. 


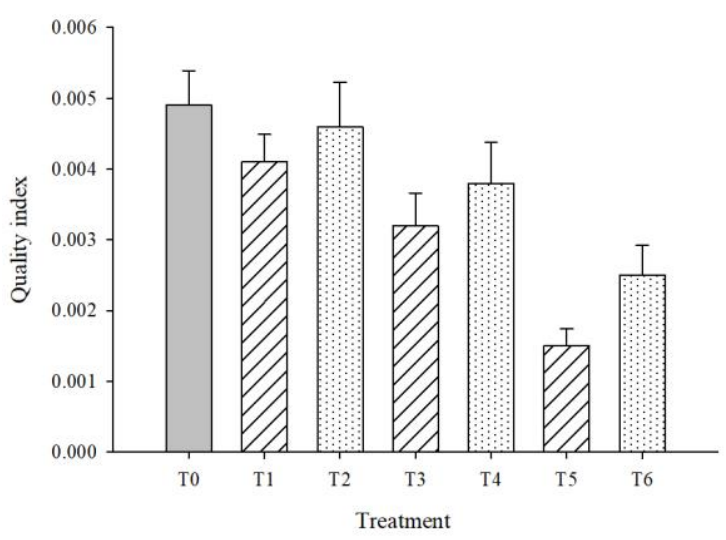

Figure 3: Effects of phosphate (Pi) on quality index of Cucumis melo in arsenic (As) condition. $\mathrm{T} 0=$ Control, $\mathrm{T} 1=2 \mathrm{ppm}$ As, $\mathrm{T} 2=2 \mathrm{ppm}$ As $+10 \mathrm{ppm} \mathrm{Pi}, \mathrm{T} 3=5 \mathrm{ppm}$ As, T4= 5ppm As + 10ppm Pi, T5=10ppm As, T6=10ppm As + 10ppm Pi.

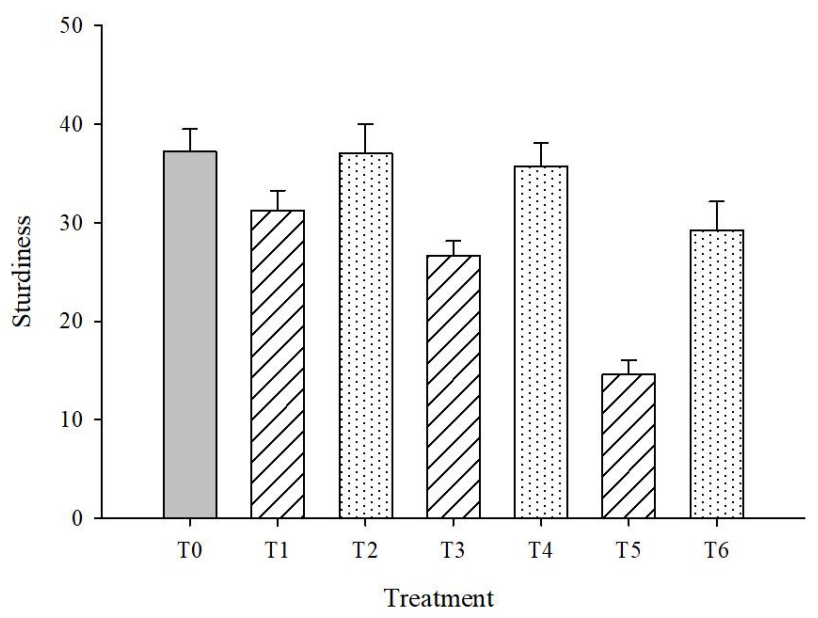

Figure 4: Phosphate (Pi) on sturdiness of Cucumis melo in arsenic (As) condition. $\mathrm{T} 0=$ Control, $\mathrm{T} 1=2 \mathrm{ppm}$ As, $\mathrm{T} 2=2 \mathrm{ppm} \mathrm{As}+10 \mathrm{ppm} \mathrm{Pi}, \mathrm{T} 3=5 \mathrm{ppm}$ As, $\mathrm{T} 4=5 \mathrm{ppm}$ As + 10ppm Pi, T5= 10ppm As, T6= 10ppm As + 10ppm Pi. 
Accumulation of As mainly occurs in the root system in plants, and in the aboveground organs to a lesser degree. Such accumulation reduces crop productivity through physiological changes in plants $[5,9,19,20,31]$. As interrupts the biochemical function of cells and severely impedes different plant metabolic processes including transpiration, respiration, photosynthesis etc., by reacting with proteins and enzymes which culminates in stunted plant growth [32]. Plants must take up sufficient amount of Pi to balance excessive As for the alleviation of As toxicity. The plants react to As exposure by increasing Pi accumulation [9, 19, 33].

This study found that, As solution culture hampers the progress of seed germination with respect to time. It also reduces the rate of seed germination, seedling growth and biomass production of the seedling of $C$. melo to varying magnitudes based on the level of As concentrations (2, 5 and 10ppm). However, addition of Pi (10 ppm) in As solution cultures (2, 5 and 10ppm respectively) offsetted the harms significantly in comparison to corresponding Pi-free As solutions. The ratio of plumule and radicle growth mostly increased with the increase of the concentration of As and the increment was further enhanced with the addition of Pi (10ppm) in As (10ppm) solution culture. These change of the growth behaviours of plant could be because plant allocates growth materials toward its plumule and radicle differently under different environmental conditions especially to well survive in the stress environmental conditions [34-37]. On the other hand, Lou-Hing et al. [38] reported that phosphate protects a rice variety- Bala from As toxicity at a lower concentration $(13.3 \mu \mathrm{M})$ of As in the solution culture. Since As is a phosphate analogue, phosphate transporters transports As which results in competition between Pi and As for uptake at the level of membrane transport. Accordingly, $\mathrm{Pi}$ is expected to reduce arsenate influx resulting ultimately into an observed increase in arsenate tolerance under phosphate addition $[19,20,39,40]$. Studies, especially in hydroponic environments, have demonstrated that non-resistant plants can be made more resistant to arsenate by raising their $\mathrm{Pi}$ status, as the $\mathrm{Pi}$ is taken more effectively compared to arsenate [5, 19, 24, 25]. Increasing cytoplasmic phosphate concentration in wheat also demonstrated the role of phosphate against As toxicity [41]. Higher phosphate 
concentration plays an important role to down-regulation of the arsenate-phosphate plasma membrane transporter and competes with arsenate for biochemical processes where arsenate substitutes for phosphate [42].

\section{Conclusion}

Our experiments showed that, presence of As in solution culture hampers the progress of seed germination with respect to time. It also reduced the rate of seed germination, seedling growth and biomass production of the seedling of C. melo at increasing magnitudes with the increasing of As concentrations. Similar trend was also continued for vigor, volume and quality indices, and also for sturdiness. However, the addition of $\mathrm{Pi}$ in As solution culture helped to minimize the negative effects of As on the progress of seed germination of this species and also enhanced the rate of seed germination, seedling growth and biomass production of seedling. Addition of $\mathrm{Pi}$ in As solution cultures offsetted the harms on vigor, volume and quality indices, and also on sturdiness significantly in comparison to corresponding Pi-free As solutions. Hence, to reduce the negative effects of As on C. melo growth and development in the laboratory, application of heightened dosage of Pi can be recommended in solution culture that contain As. This similar approach might also be applicable in the field.

\section{References}

[1] M. Malek, M. O. Islam, M. Mamtazul, and M. K. Sultan; Bangladesh Journal of Agricultural Research, 2012, 37(3), 465-472.

[2] G. E. Lester, and D. M. Hodges; Postharvest Biology and Technology, 2008, 48, 347-354.

[3] K. Foord, and J. MacKenzie; Growing melons (cantaloupe, watermelon, honeydew) in Minnesota home gardens. https://www.extension.umn.edu/garden/ yardgarden/fruit/growing-melons-in-minnesota-home-gardens, 2017.

[4] A. A. Meharg, and M. A. Rahman; Environmental Science and Technology, 2003, 37, 229-234.

[5] B. M. Khan, A. H. Afrin, and M. M. Hossain; International Journal of Rural Development, Environment and Health Research, 2018, 2(1), 48-55.

[6] A. A. Guevara-Garcia, F. P. Lara, L. K. Juarez, and L. R. Herrera-Estrella; eLS, 2017, 1-9. 
Effects of Phosphate (Pi) on Seed Germination and Seedling Growth of Cucumis melo L. in Arsenic Supplemented Medium

[7] A. A. Babst-Kostecka, P. Waldmann, H. Frerot, and P. Vollenweider; Environmental and Experimental Botany, 2016, 127, 1-13.

[8] M. Singh, J. Kumar, S. Singh, V. P. Singh, S. M. Prasad, and M. P. V. V. B. Singh; Biochemistry \& Pharmacology, 2015, 4, 161. doi:10.4172/2167-0501.1000161.

[9] R. Leimu, and M. Fischer; PLOS ONE, 2008, 3(12): e4010. doi:10.1371/journal.pone.0004010.

[10] F. Sanal, G. Şeren, and U. Guuner; Bulletin of Environmental Contamination and Toxicology, 2014, 92(4), 483-489.

[11] W. J. Fitz, and W. W. Wenzel; Journal of Biotechnology, 2002, 99(3), 259-278.

[12] S. Sultana, M. H. Rashid, and S. M. I. Huq; Bangladesh Journal of Scientific Research, 2015, 28(2), 171-180.

[13] A. A. Carbonell-Barrachina, F. Burlo, A. Burgos-Hernandez, E. Lopez, and J. Mataix; Scientia Horticulturae, 1997, 71, 167-176.

[14] I. Chaturvedi; Journal of Central European Agriculture, 2006, 7(1), 31-40.

[15] B. K. Mishra, C. S. Dubey, D. P. Shukla, P. Bhattacharya, and A. L. Usham: Environmental Earth Sciences, 2014, 72(9), 3281-3291.

[16] B. E. Hettick; Arsenic in muskmelon (Cucumis melo) plants and wine in West Texas, 2016. https://ttu-ir.tdl.org/ttu-ir/handle/2346/68091, 2017.

[17] H. Marschner; Mineral nutrition of higher plants, $2^{\text {nd }}$ ed. Academic press, Inc., London, 1995.

[18] K. G. Raghothama; Annual Review of Plant Physiology and Plant Molecular Biology, 1999, 50, 665-693.

[19] B. M. Khan, C. Deacon, C. Meharg, G. Norton, D. Johnson, and A. A. Meharg; Environmental and Experimental Botany, 2013, 96, 43-51.

[20] C. Meharg, B. Khan, G. Norton, C. Deacon, D. Johnson, R. Reinhardt, B. Huettel, and A. A. Meharg; New Phytologist, 2014, 201, 144-154.

[21] A. A. Meharg, and M. R. Macnair; Journal of Experimental Botany, 1992a, 43, 519-524.

[22] D. C. Adriano; "Trace Elements in Terrestrial Environments. Biogeochemistry, Bioavailability and Risks of Metals", Springer, New York, 2001, 796.

[23] F. J. Peryea; HortScience, 1998, 33, 826-829.

[24] A. A. Meharg, and M. R. Macnair; Heredity, 1992b, 69, 336-341.

[25] D. A. Lee, A. Chen, and J. I. Schroeder; The Plant Journal, 2003, 35, 637-646. 
[26] F. T. Bonner; In S. K. Kamra, and R. D. Aylin (Eds). "Proceeding of the International Symposium on Forest Seed Problems in Africa', 1987, 53-61.

[27] D. R. Hoagland, and D. I. Arnon; California Agricultural Experiment Station Circular, 1950, 347, 1-32.

[28] A. Abdul-Baki, J. D. Anderson; Crop Science, 1973, 13, 630-633.

[29] G. E. Hatchell; In E. Shoulders (Ed). "Proceedings of the $3^{\text {rd }}$ Biological Southern Research Conference", 1985, 395-402.

[30] A. Dickson, A. L. Leaf, and J. F. Hosner; Forestry Chronicle, 1960, 36, 10-13.

[31] N. Stoeva, M. A. Berova, and Z. Zlatev; Biologia Plantarum, 2005, 49, 293-296.

[32] A. A. Meharg, and J. Hartley-Whitaker; New Phytologist, 2002, 154(1), 29-43.

[33] F. Burlo, I. Guijarro, A. A. C. Barrachina, and D. Vlaero; Journal of Agricultural and Food Chemistry, 1999, 47, 1247-1253.

[34] J. O. Hill, R. J. Simpson, A. D. Moore, and D. F. Chapman; Plant and Soil, 2006, 286, 7-19.

[35] H. Malhotra, Vandana, S. Sharma and R. Pandey; In M. Hasanuzzaman, M. Fujita, H. Oku, K. Nahar, B. Hawrylak-Nowak (Eds) "Plant Nutrients and Abiotic Stress Tolerance", 2018, 171-190. DOI: 10.1007/978-981-10-9044-8_7.

[36] M. Razaq, P. Zhang, H-I. Shen, and Salahuddin; PLOS ONE, 2017, 12(2), 1-13. https://doi.org/10.1371/journal.pone.0171321,2018.

[37] M. Irfan, M. Abbas, J. A. Shah, N. Depar, M. Y. Memon, and N. A. Sial; Eurasian Journal of Soil Science, 2019, 8(1), 17-26.

[38] D. Lou-Hing, B. Zhang, A. H. Price, and A. A. Meharg; Environmental and Experimental Botany, 2011, 72, 47-52.

[39] C. I. Ullrich-Eberius, A. Sanz, and and A. J. Novacky; Journal of Experimental Botany, 1989, 40(210), 119-128.

[40] A. A. Meharg, and M. R. Macnair; New Phytologist, 1990, 116, 29-35.

[41] A. A. Meharg; Reviews on Environmental Contamination and Toxicology, 1994, 138, 21-48.

[42] M. Pigna, V. Cozzalino, A. Violante, and A. A. Meharg; Water, Air, and Soil Pollution, 2009, 197, 371-380.

The Chittagong Univ. J. Sc. Vol. 43(1), 2021 